\title{
Machine Learning Application for Rupture Risk Assessment in Small-Sized Intracranial Aneurysm
}

\author{
Heung Cheol Kim ${ }^{1,+}$, Jong Kook Rhim ${ }^{2,+}$, Jun Hyong Ahn ${ }^{3}$, Jeong Jin Park ${ }^{4}$, Jong Un Moon ${ }^{5}$, \\ Eun Pyo Hong ${ }^{6}{ }^{\oplus}$, Mi Ran Kim ${ }^{7}$, Seung Gyu Kim ${ }^{7}$, Seong Hwan Lee ${ }^{7}$, Jae Hoon Jeong ${ }^{7}$, \\ Sung Won Choi ${ }^{7}$ and Jin Pyeong Jeon ${ }^{3,8,9, *}$ \\ 1 Department of Radiology, Hallym University College of Medicine, Chuncheon 24252, Korea; \\ khc@hallym.or.kr \\ 2 Department of Neurosurgery, Jeju National University College of Medicine, Jeju 63241, Korea; \\ pedineur@daum.net \\ 3 Department of Neurosurgery, Hallym University College of Medicine, Chuncheon 24252, Korea; \\ sparkahn@naver.com \\ 4 Department of Neurology, Konkuk University Medical Center, Seoul 05030, Korea; medicalstory@gmail.com \\ 5 Department of Neurosurgery, National Medical Center, Seoul 04564, Korea; nsdocmoon@gmail.com \\ 6 Molecular Neurogenetics Unit, Center for Genomic Medicine, Massachusetts General Hospital, Boston, \\ MA 02114, USA; ephong0305@gmail.com \\ $7 \quad$ Buzzpole Inc., Seoul 04781, Korea; ran@buzzpole.com (M.R.K.); seung@buzzpole.com (S.G.K.); \\ usim@buzzpole.com (S.H.L.); jman@buzzpole.com (J.H.J.); wociety@buzzpole.com (S.W.C.) \\ 8 Institute of New Frontier Stroke Research, Hallym University College of Medicine, Chuncheon 24252, Korea \\ 9 Genetic and Research Inc., Chuncheon 24252, Korea \\ * Correspondence: jjs6553@daum.net; Tel.: +82-33-240-5171; Fax: +82-33-240-9970 \\ + Contributed equally to this work.
}

Received: 22 April 2019; Accepted: 13 May 2019; Published: 15 May 2019

\begin{abstract}
The assessment of rupture probability is crucial to identifying at risk intracranial aneurysms (IA) in patients harboring multiple aneurysms. We aimed to develop a computer-assisted detection system for small-sized aneurysm ruptures using a convolutional neural network (CNN) based on images of three-dimensional digital subtraction angiography. A retrospective data set, including 368 patients, was used as a training cohort for the $\mathrm{CNN}$ using the TensorFlow platform. Aneurysm images in six directions were obtained from each patient and the region-of-interest in each image was extracted. The resulting CNN was prospectively tested in 272 patients and the sensitivity, specificity, overall accuracy, and receiver operating characteristics (ROC) were compared to a human evaluator. Our system showed a sensitivity of $78.76 \%$ (95\% CI: $72.30 \%-84.30 \%)$, a specificity of $72.15 \%$ (95\% CI: $60.93 \%-81.65 \%$ ), and an overall diagnostic accuracy of $76.84 \%$ (95\% CI: $71.36 \%-81.72 \%$ ) in aneurysm rupture predictions. The area under the ROC (AUROC) in the CNN was 0.755 (95\% CI: $0.699 \%-0.805 \%$ ), better than that obtained from a human evaluator (AUROC: $0.537 ; p<0.001$ ). The CNN-based prediction system was feasible to assess rupture risk in small-sized aneurysms with diagnostic accuracy superior to human evaluators. Additional studies based on a large data set are necessary to enhance diagnostic accuracy and to facilitate clinical application.
\end{abstract}

Keywords: intracranial aneurysm; convolutional neural network; subarachnoid hemorrhage

\section{Introduction}

The prevalence of intracranial aneurysms (IA) has been reported to be as high as 3.2 percent [1,2]. However, the rate of subarachnoid hemorrhage (SAH) due to aneurysm rupture is less than $2 \%$, which means that only a small number of patients experience rupture events [3]. Nevertheless, if a rupture 
occurs, mortality rates up to $57 \%$ within the first six months have been reported $[4,5]$. Accordingly, the identification of unruptured intracranial aneurysms (UIA) and the risk assessment of future rupture events are important in treatment planning. In clinical circumstances, aneurysm location and size are the main factors influencing UIA treatment. The annual rupture rate of aneurysms in the internal carotid artery (ICA), the posterior communicating (P-com) artery, and the middle cerebral artery (MCA) is $1.6 \%$. In contrast, UIA patients with aneurysms in the anterior cerebral artery (ACA), including the main anterior communicating (A-com) artery and the posterior cerebral artery, had higher annual rupture rates of up to $1.9 \%$ and $4 \%$, respectively [6,7]. Size is an important determinate for rupture risk. Aneurysm sizes of $7 \mathrm{~mm}$ and greater have been identified as risk factors increasing lifelong rupture risk [8]. Due to technical advances and more detailed radiologic findings, such as irregular aneurysm walls, multi-lobulation, and hemodynamic patterns focusing on aneurysm wall shear stress (WSS) have gained attention [9]. However, many uncertainties remain regarding rupture risk, particularly in small saccular aneurysms less than $7 \mathrm{~mm}$ in size.

Recently, a deep learning algorithm using a convolutional neural network (CNN) has been increasingly applied to a broad variety of medical imaging modalities. Aubreville et al. [10] reported that a CNN showed a mean accuracy of $88.3 \%$ (sensitivity $86.6 \%$ and specificity $90 \%$ ) for the automatic classification of cancerous tissue in confocal laser endomicroscopic image sequences of the oral cavity. Esteva et al. [11] also demonstrated that a CNN using only pixels and disease labels as input variables provided detection of skin cancer comparable to dermatologists. A CNN has also been used for automated aneurysm detection [12] and measurement in patients with IA [13]. Nakao et al. [12] reported that aneurysm detection rate by the $\mathrm{CNN}$ based on a maximum intensity projection (MIP) algorithm of magnetic resonance angiography (MRA) was $94.2 \%$, with 2.9 false positives per case. In addition, the CNN measured aneurysm areas correctly more often than a radiologist [13].

Beyond these findings, another important issue for $\mathrm{CNN}$ application is to distinguish between aneurysms that have ruptured, and those that have not. Rupture status can be easily diagnosed by hemorrhage patterns on the computed tomography (CT), however, multiple aneurysms have been reported in up to $33.5 \%$ of patients $[2,14,15]$. In addition, in patients with multiple aneurysms, $31 \%$ harbored three or more (120 of 387) aneurysms. Since inaccurate identification of a ruptured aneurysm can lead to re-bleeding events [16], accurate assessment of a rupture is essential. Therefore, we have applied a CNN to images of three-dimensional (3D) digital subtraction angiography (DSA) to evaluate the rupture status in patients with small-sized aneurysms of the anterior circulation.

\section{Materials and Methods}

\subsection{Study Population}

This study included retrospective data collection and a prospective study in patients harboring IA at multiple centers between January 2012 and December 2018. Patients with a clinical diagnosis and neuroimaging findings of aneurysm morphology were recruited. The inclusion criteria in this study were: (1) adult patients over 18 years old, (2) a diagnosis of saccular aneurysms, (3) an IA located on the anterior circulation, and (4) small aneurysms with less than $7 \mathrm{~mm}$ maximal diameters. The exclusion criteria were: (1) a diagnosis of non-saccular aneurysms, such as fusiform or dissecting aneurysms; (2) aneurysms due to trauma or infection; (3) an IA on the posterior circulation; and (4) patients with multiple aneurysms. A retrospective data set composed of IA patients who underwent DSA between January 2012 and December 2016 was used as a training cohort for the CNN and the AlexNet architecture. The resulting CNN was then prospectively tested in IA patients who underwent 3D-DSA (3D-digital subtraction angiography) from January 2017 to December 2018 [17]. The aims of this investigation were to develop an automatic classification system to distinguish ruptured from unruptured aneurysms using 3D-DSA images, and to demonstrate the feasibility of the system using a training and validation test set. The clinical and radiologic data were reviewed by two readers (KHC and JPJ). This study was approved by the Institutional Review Board (No. 2018-11-008). 


\subsection{Image Acquisition and Data Processing}

DSA was conducted with the Allura Xper FD 20/20 (Philips Medical System, Best, The Netherlands) biplane system and the Axiom Artis zee (Siemens Healthcare, Erlangen, Germany) in conjunction with standard injection protocols [18,19]. For 3D-DSA, a total of $20 \mathrm{~mL}$ of Iodixanol 320 (Visipaque, GE Healthcare, Chicago, IL, USA) was administered at $4 \mathrm{~mL} / \mathrm{s}$ for 12 seconds and 121 projections were obtained in $240^{\circ}$ within 4 seconds to construct 3D images. There was a 1.5 second time delay for contrast filling in the Allura Xper FD 20/20 system. For the Axiom Artis zee, a total of $18 \mathrm{~mL}$ of Pamiray 250 contrast agent (Iopamidol, Dong-kook Pharmaceutical, Seoul) was administered with a tube rotation time of 4.5 seconds. The images were reconstructed using a $256 \times 256$ matrix. Post-processing of 3D-DSA was done in an independent workstation equipped with InSpace 3D software [18-20]. Aneurysm images in six directions (anteroposterior, posteroanterior, both laterals, superoinferior and inferosuperior) were obtained. Regions-of-interest (ROIs) selected by the neuroradiologist which included the aneurysm were then applied to each image, as seen in Figure 1.

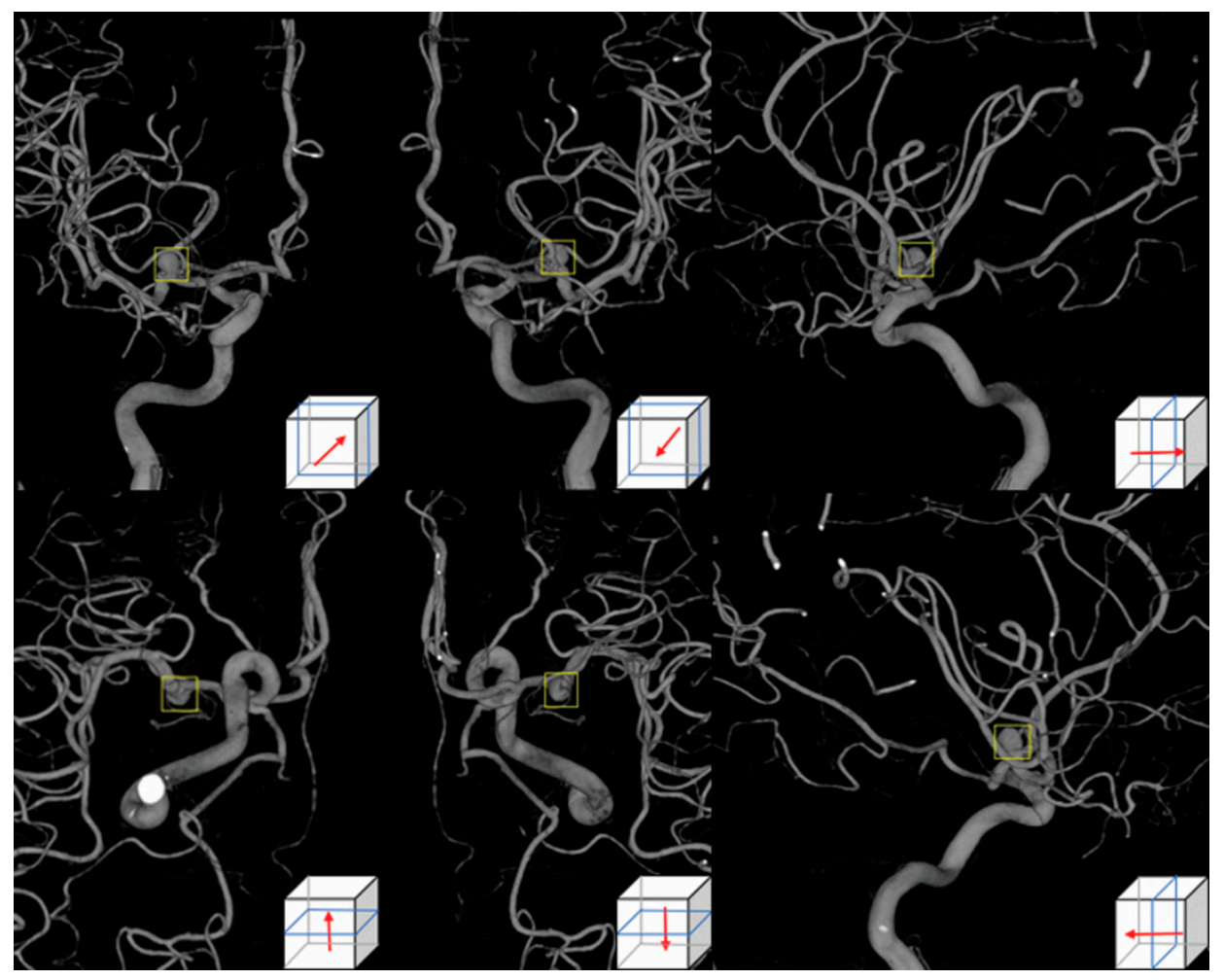

Figure 1. Aneurysm images using 3D-digital subtraction angiography in six directions, anteroposterior, posteroanterior, left lateral, superoinferior, inferosuperior, and the right lateral side. A user-selected region-of-interest shown as a yellow square was applied to each image.

\subsection{Neural Network Architecture for Aneurysm Images}

A CNN with an Alexnet_v2 architecture was used. For the third through the fifth convolution layers, $3 \times 3$ sized filters were used. For the second layer and the first layer, $5 \times 5$ and $11 \times 11$ sized filters were used, respectively. The system received three channel $224 \times 224$ sized input images extracted with a diameter of 65 pixels, consisting of three max pooling layers, two drop-out layers, three fully connected layers, and five convolution layers as seen in Figure 2. The model was implemented on a TensorFlow API (Google Inc, Mountain View, CA, USA). Data sets were completely balanced (on categories) and resized to $224 \times 224$ as train data sets. In addition, data augmentation was performed. We flipped each piece of data horizontally and vertically. Accordingly, we were able to get four times more trained images in total compared to the original trained images. All parameters were 
trained from scratch using the Adam optimization method with a batch size of 20 on the Geforce RTX 2080 GPU (NVIDIA, Santa Clara, CA, USA). The learning rate was set to be $5 \times 10^{-7}$ and the drop-out rate was 0.5. The Adam optimizer was set with the default parameters. Ruptured aneurysms were defined as those with an expected rupture risk of $\geq 50 \%$ among the aneurysm images after discussion.

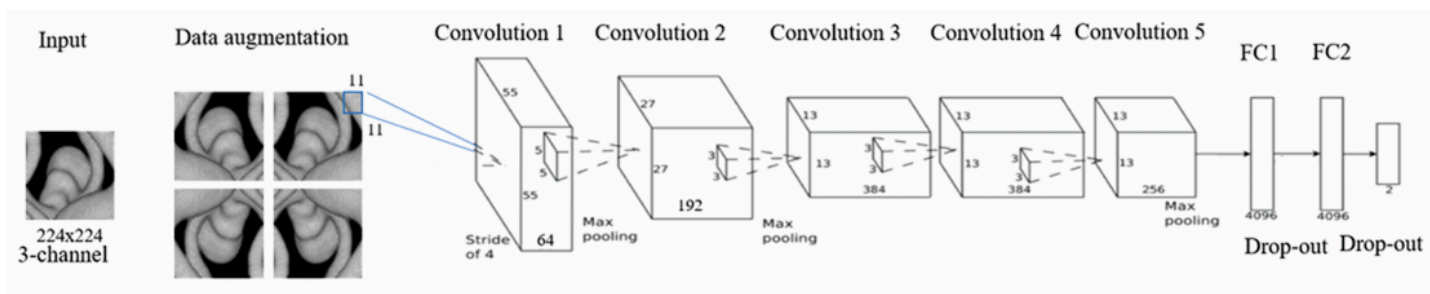

Image patch

Trained filter of the first convolution layer

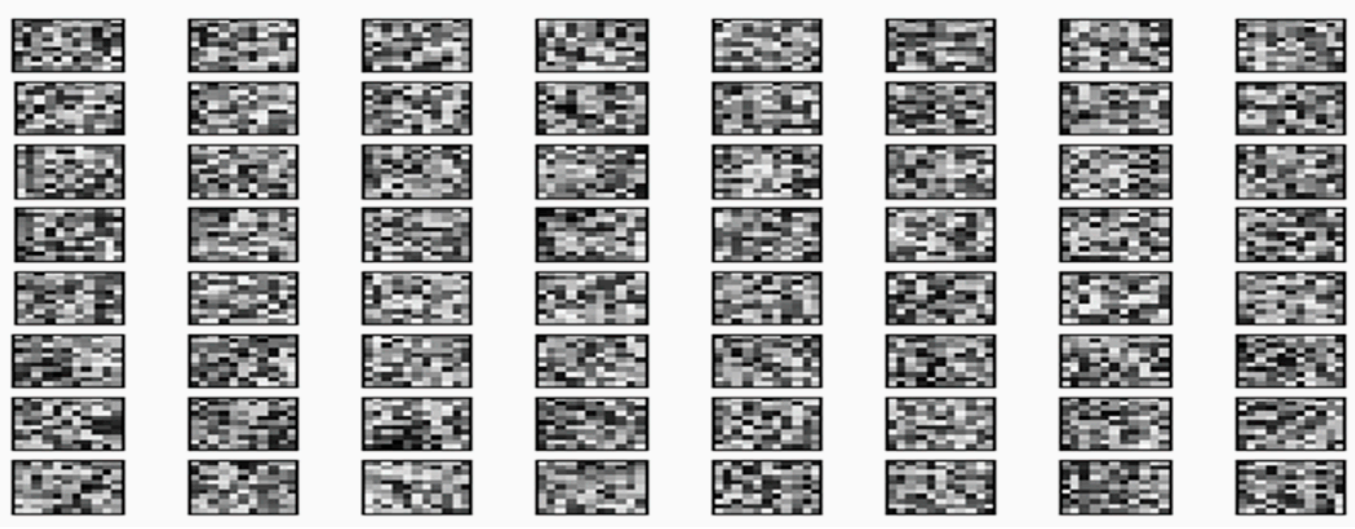

Figure 2. The proposed convolutional neural network consisting of convolutional layers, max-pooling layers, fully-connected layers, drop-out layers, and final layers of classification. The number of filters, the connections in each layer, and the first layer of the learned convolutional kernel are described above. FC, fully-connected.

\subsection{Statistical Analyses}

Descriptive results were presented as the number of subjects (percentage) for both discrete and categorical variables, and as the mean and standard deviation (SD). A two-by-two table was generated to assess the sensitivity, specificity, and overall accuracy of the results [18]. Prospective data of the test cohort obtained by the $\mathrm{CNN}$ or by the human evaluator were analyzed using receiver operating characteristics (ROC). Any $p$-values less than 0.05 were considered statistically significant. The analysis was conducted using SPSS version 19 (IBM, Armonk, NY, USA) and MedCalc (www.Medcalc.org).

\section{Results}

\subsection{Clinical Characteristics of the Enrolled Patients}

A total of 640 patients with IA were included. There were 368 patients in the retrospective training data set and 272 patients in the prospective test group as seen in Table 1. There are no differences in the number of females, incidence of hypertension, diabetes mellitus, hyperlipidemia, or smoking between the two groups as seen in Table 1. The number of SAHs present was 244 (66.3\%) in the training cohort and $191(70.2 \%)$ in the test cohort. The number of aneurysm locations did not reach statistical significance between the two groups $(p=0.265)$. 
Table 1. Comparison of clinical and radiologic characteristics between the training and test cohorts.

\begin{tabular}{cccc}
\hline Variables & $\begin{array}{c}\text { Training Cohort } \\
(\boldsymbol{n}=\mathbf{3 6 8})\end{array}$ & $\begin{array}{c}\text { Test Cohort } \\
(\boldsymbol{n}=\mathbf{2 7 2})\end{array}$ & $\boldsymbol{p}$-Value \\
\hline Clinical findings & $175(47.6 \%)$ & $145(53.3 \%)$ & 0.150 \\
Female & $57.8 \pm 14.4$ & $55.8 \pm 16.3$ & 0.101 \\
Age, years & $89(24.2 \%)$ & $72(26.5 \%)$ & 0.510 \\
Hypertension & $37(10.1 \%)$ & $28(10.3 \%)$ & 0.921 \\
Diabetes mellitus & $38(10.3 \%)$ & $21(7.7 \%)$ & 0.260 \\
Hyperlipidemia & $44(12.0 \%)$ & $27(9.9 \%)$ & 0.419 \\
Smoking & $172(46.7 \%)$ & $127(46.7 \%)$ & 0.990 \\
Radiologic findings & $244(66.3 \%)$ & $193(71.0 \%)$ & 0.294 \\
Lesion side, left & $5.3 \pm 1.0$ & $5.2 \pm 1.1$ & 0.231 \\
SAH presentation & & & \\
Size (mm) & $123(33.4 \%)$ & $96(35.3 \%)$ & 0.265 \\
Territory location & $133(36.1 \%)$ & $82(30.1 \%)$ & \\
Anterior cerebral artery & $112(30.5 \%)$ & $94(34.6 \%)$ & \\
Middle cerebral artery & & \\
Internal cerebral artery & & & \\
\hline
\end{tabular}

Subarachnoid hemorrhage (SAH). Data are shown as the numbers of subjects (percentage) for discrete and categorical variables and mean \pm standard deviation.

\subsection{Accuracy of CNN for the Diagnosis of Ruptured Aneurysm}

In the test cohort, the CNN demonstrated a sensitivity of $78.76 \%$ (95\% CI: $72.30 \%-84.30 \%$ ), a specificity of $72.15 \%$ (95\% CI: $60.93 \%-81.65 \%)$, and an accuracy of $76.84 \%(95 \%$ CI: $71.36 \%-81.72 \%)$ to predict ruptured aneurysms, as seen in Table 2. The AUROC for the CNN was 0.755 (95\% CI: $0.699 \%-0.805 \%)$ and was significantly better $(p<0.001)$ than that of the human evaluator (AUROC $=0.537 ; 95 \%$ CI: $0.476 \%-0.598 \%$ ), as seen in Figure 3. We further evaluated accuracy according to aneurysm locations. For IA patients with ACA aneurysms, the CNN demonstrated a sensitivity of $86.36 \%$ (95\% CI: $75.69 \%-93.57 \%$ ), a specificity of $36.67 \%$ (95\% CI: $19.93 \%-56.14 \%$ ), and an accuracy of 70.83 percent (95\% CI: $60.67 \%-79.67 \%$ ). For MCA aneurysms, the CNN showed a sensitivity of $85.42 \%$ (95\% CI: $72.24 \%-93.93 \%$ ) and a specificity of 79.41 percent $(95 \%$ CI: $62.10 \%-91.30 \%)$. Regarding ICA aneurysms, the sensitivity and specificity were $90.00 \%$ (95\% CI: 79.49\%-96.24\%) and $55.88 \%$ (95\% CI: $37.89 \%-72.81 \%)$, respectively.

Table 2. Accuracy of the CNN to detect ruptured or unruptured intracranial aneurysms in the test cohort ${ }^{\mathrm{a}}$.

\begin{tabular}{cccc}
\hline & Ruptured & Unruptured & Total \\
\hline Ruptured & 152 & 22 & 176 \\
Unruptured & 41 & 57 & 96 \\
Total & 193 & 79 & 272 \\
\hline
\end{tabular}

${ }^{\mathrm{a}}$ The numbers given are patients. 


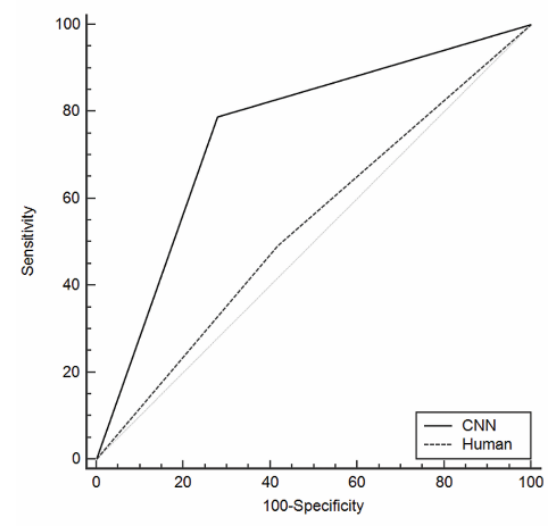

\begin{tabular}{cccc}
\hline Criterion & AUROC & $\mathbf{9 5 \%}$ CI & $p$-Value \\
\hline CNN $(>0)$ & 0.755 & $0.699-0.805$ & $<0.001$ \\
Human $(>0)$ & 0.537 & $0.476-0.598$ & \\
\hline
\end{tabular}

Figure 3. Receiver operating characteristics (ROC) curve according to detections by the convolutional neural network $(\mathrm{CNN})$ and a human evaluator. The difference between areas is $0.163(p<0.001)$. Area under the ROC (AUROC) curve.

\section{Discussion}

To the best of our knowledge, this is the first study that applied a CNN to assess rupture risk in small-sized aneurysms. Overall, the CNN had a sensitivity of $78.76 \%$ (95\% CI: $72.30 \%-84.30 \%$ ) and a specificity of $72.15 \%$ (95\% CI: $60.93 \%-81.65 \%)$ for predicting ruptures in small-sized aneurysms. In addition, the CNN showed higher AUROC than experienced human evaluators $(0.755$ and 0.537 , respectively).

Anatomical and hemodynamic characteristics have been widely studied to identify morphological parameters associated with IA rupture risk. Aneurysms are classified into several groups by their size: small $(<7 \mathrm{~mm})$, medium $(7-12 \mathrm{~mm})$, large $(13-24 \mathrm{~mm})$, and giant $(\leq 25 \mathrm{~mm})$ [21]. The first international study of unruptured intracranial aneurysms (ISUIA) reported that the rupture risk in patients with IA with a size of less than $10 \mathrm{~mm}$, was less than $0.05 \%$ per year [22]. The second study of ISUIA also reported that IA patients who had aneurysms with a size of less than $7 \mathrm{~mm}$ without previous SAH histories had a $\%$ 5-year cumulative rupture rate in anterior circulation aneurysms of the ACA, MCA, and ICA, as well as a $2.5 \%$-year cumulative rupture rate in posterior circulation and P-com aneurysms [23,24]. In contrast, patients with medium-sized UIAs in anterior circulation aneurysms, with the exception of cavernous aneurysms, had rupture rates of $1.5 \%$ and of $3.4 \%$ in posterior circulation and P-com aneurysms, respectively. Accordingly, it is reasonable to observe small aneurysms using repeat radiologic tests in patients without risk factors, such as prior SAH history, the presence of P-com or daughter sacs [25]. However, very small aneurysms $(<5 \mathrm{~mm})$ can rupture. Dolati et al. [24] reported that the rupture rate of aneurysms $<5 \mathrm{~mm}$ was $37 \%$ among SAH patients. In hemodynamic studies, lower WSS and high oscillatory shear index (OSI) were associated with aneurysm ruptures (AUC $=0.85,95 \%$ CI: 0.78-0.93) [26]. The authors suggested that increased permeability of the endothelium caused by enhanced endothelial adhesion molecules in lower WSS and high OSI could lead to aneurysm formation and degradation of the wall [27,28]. However, Hassan et al. [29] reported that higher WSS was related to IA growth and rupture in wide-necked aneurysms. These conflicting results suggested that higher WSS may initiate aneurysm formation and that lower WSS may aggravate aneurysm growth and subsequent rupture [30].

In recent years, CNNs have been used for various neuroradiology imaging findings, focusing on the development of efficient automatic diagnoses of solid tumors and vascular diseases. Laukamp et al. [31] 
reported that the multiparametric deep learning model (DLM) accurately diagnosed meningiomas in 55 out of 56 cases which correlated strongly (0.81) with manual segmentation measurements of tumor volumes. Stember et al. [13] demonstrated the highly accurate detection of IAs (85/86, 98.8\%) using a CNN in conjunction with an MRA. In their study, 2D-MIP images were used for validation. Accordingly, some aneurysms located on or near tortuous parent arteries led to focal localization [13]. Nakao et al. [12] also reported the high accuracy of an automatic CNN system $(94.2 \%, 98 / 104)$ to detect IAs. They reconstructed MRA images with a $0.469 \mathrm{~mm}$ isotropic voxel size and extracted each voxel inside or outside the arterial region from a volume of interest (VOI) along nine axes of MIP images [12]. In our study, we focused on predicting aneurysm rupture, not on diagnosis of the aneurysm itself. Although MRAs showed a high pooled sensitivity of 95\% (95\% CI: $89 \%-98 \%$ ) in IA detection [32], DSA is widely accepted as a key radiologic examination used to identify delicate aneurysm morphologies and to set treatment plans. In addition, 3D-DSA provides clearer information on aneurysm characteristics and their relationship to nearby arteries than standard 2D or rotational DSA [33]. We collected DSA images in six directions in each patient and extracted images from the selected ROI. ROI images were then trained with the CNN classifier using an Alexnet_v2 architecture as seen in Figure 2. The output layer was a single unit and each image was assigned 0 or 1 , according to the probability of being a ruptured aneurysm [12]. In the test cohort, the decision was made by the rupture rate in six images, whereby $\geq 50 \%$ indicated a positive finding. Compared to a neuro-interventionist, the $\mathrm{CNN}$ classifier showed better diagnostic accuracy to predict aneurysm rupture (AUROC difference $=0.218 ; p<0.001$ ).

Our system can be helpful to identify culprit aneurysms which can lead to SAH, particularly in patients harboring multiple aneurysms. The incidence of multiple aneurysms has been reported to be over $30 \%[2,15]$. Nehls et al. [15] reported the common locations of multiple aneurysms in the order of P-com (21.5\%), A-com (12\%), and the ophthalmic artery (11\%). Although MCA was the lowest site for rupture events in their study [15], identification of the culprit aneurysm was based on the distribution of the hemorrhage and the aneurysm's morphologies. Nevertheless, the results could be arbitrary and largely based on the neurosurgeon's experience. In our study, the AUROC of an experienced neuro-interventionist was 0.537 (95\% CI: $0.476 \%-0.598 \%$ ), indicating poor diagnostic accuracy for the detection of ruptured aneurysms. Accordingly, our CNN classifier enhanced the diagnostic accuracy for culprit aneurysms.

In this study, we performed additional trials using different combinations. First, we switched the CNN network to a Residual neural network (ResNet) 50 under the same conditions. We then used a pretrained ImageNet ResNet50 model without data augmentation. Second, we applied a histogram of oriented gradients (HOG) method on our training images without data augmentation trained with Alexnet_v2. Overall diagnostic accuracy was $60.53 \%$ in ResNet-50 and $65.17 \%$ in HOG + Alexnet, showing lower than Alexnet_v2 with data augmentation.

There were some limitations to this study. First, we only included anterior circulation aneurysms. Anterior circulation aneurysms are more prevalent than posterior circulation aneurysms, at an approximate ratio of 7:3. In addition, the morphometric factors between anterior and posterior circulation aneurysms are different [34]. Tykocki et al. [34] reported that the aspect ratio and parent artery size were significantly different between patients with anterior and posterior circulation aneurysms. Accordingly, further studies to assess aneurysm rupture are warranted for aneurysms of the posterior circulation. Second, differences in angiographic machines were not considered in the interpretation of our results. Moreover, the criteria for choosing radiologic tests for IA are somewhat different. Third, we applied user-selected ROI around the aneurysm, therefore, the system was not a fully automatic classifier. A previous automatic detection system [12] also determined VOI empirically for image extraction. The CNN with an Alexnet_v2 network used in this study trains faster compared to earlier network models using sigmoid function. However, compared to other network architectures such as Visual geometry group (VGG)-16, the Alexnet network retains more information of unrelated background in the final convolutional layer [35]. Accordingly, we applied user-selected ROI around the aneurysm. Our results showed a sensitivity of $78.76 \%$, a specificity of $72.15 \%$, and an accuracy of 
$76.84 \%$ in predicting ruptured aneurysm. Nevertheless, further studies are needed to compare deep neural networks in large datasets and automatic ROI detection of aneurysms.

\section{Conclusions}

We proposed a computer-assisted detection system to assess aneurysm rupture probability using a CNN based on 3D-DSA images. Our system was feasible to detect the rupture of small-sized aneurysms and demonstrated superior diagnostic accuracy compared to human evaluators. Future studies, based on a large data-set including posterior circulation aneurysms, are required to increase the diagnostic accuracy and to facilitate its clinical application.

Author Contributions: Conceptualization, J.J.P.; methodology and formal analysis, M.R.K., S.G.K., S.H.L., J.H.J., S.W.C., J.H.A., J.J.P., J.U.M., and E.P.H.; writing-original draft preparation, H.C.K., and J.K.R.; supervision, J.P.J.

Funding: This research was supported by the Korea Technology and Information Promotion Agency for SMEs (S-2693922) and the Hallym University Research Fund (HURF-2019-22).

Conflicts of Interest: The authors declare no conflict of interest.

\section{Abbreviations and Acronyms}

ACA, Anterior cerebral artery; A-com, Anterior communicating artery, AUROC, Area under the receiver operating characteristics; CI, Confidence interval; CNN, Convolutional neural network; CT, Computed tomography; DLM, Deep learning model; DSA, Digital subtraction angiography; HOG, Histogram of oriented gradients; IA, Intracranial aneurysm; ICA, Internal carotid artery; ISUIA, International study of unruptured intracranial aneurysms; MCA, Middle cerebral artery; MIP, Maximum intensity projection; MRA, Magnetic resonance angiography; OSI, Oscillatory shear index; P-com, Posterior communicating artery; ROC, Receiver operating characteristics; ROI, Regions-of-interest; ResNet, Residual neural network; SAH, Subarachnoid hemorrhage; SD, Standard Deviation; 3D, Three-dimension; UIA, Unruptured intracranial aneurysm; VGG, Visual geometry group; VOI, Volume of interest; WSS, Wall shear stress.

\section{References}

1. Vlak, M.H.; Algra, A.; Brandenburg, R.; Rinkel, G.J. Prevalence of unruptured intracranial aneurysms, with emphasis on sex, age, comorbidity, country, and time period: A systematic review and meta-analysis. Lancet Neurol. 2011, 10, 626-636. [CrossRef]

2. Thompson, B.G.; Brown, R.D., Jr.; Amin-Hanjani, S.; Broderick, J.P.; Cockroft, K.M.; Connolly, E.S., Jr.; Duckwiler, G.R.; Harris, C.C.; Howard, V.J.; Johnston, S.C.; et al. Guidelines for the management of patients with unruptured intracranial aneurysms: A guideline for healthcare professionals from the american heart association/american stroke association. Stroke 2015, 46, 2368-2400. [CrossRef] [PubMed]

3. Katati, M.J.; Santiago-Ramajo, S.; Perez-Garcia, M.; Jofré, M.M.S.; Vilar-Lopez, R.; Coin-Mejias, M.A.; Caracuel-Romero, A.; Arjona-Moron, V. Description of quality of life and its predictors in patients with aneurysmal subarachnoid hemorrhage. Cerebrovasc. Dis. 2007, 24, 66-73. [CrossRef] [PubMed]

4. Lantigua, H.; Ortega-Gutierrez, S.; Schmidt, J.M.; Lee, K.; Badjatia, N.; Agarwal, S.; Claassen, J.; Connolly, E.S.; Mayer, S.A. Subarachnoid hemorrhage: Who dies, and why? Crit. Care 2015, 19, 309. [CrossRef] [PubMed]

5. Bonita, R.; Thomson, S. Subarachnoid hemorrhage: Epidemiology, diagnosis, management, and outcome. Stroke 1985, 16, 591-594. [CrossRef] [PubMed]

6. Juvela, S. Natural history of unruptured intracranial aneurysms: Risks for aneurysm formation, growth, and rupture. Acta Neurochir. Suppl. 2002, 82, 27-30. [PubMed]

7. Clarke, G.; Mendelow, A.D.; Mitchell, P. Predicting the risk of rupture of intracranial aneurysms based on anatomical location. Acta Neurochir. (Wien) 2005, 147, 259-263. [CrossRef]

8. Korja, M.; Lehto, H.; Juvela, S. Lifelong rupture risk of intracranial aneurysms depends on risk factors: A prospective finnish cohort study. Stroke 2014, 45, 1958-1963. [CrossRef] [PubMed]

9. Jeon, J.S.; Ahn, J.H.; Huh, W.; Son, Y.J.; Bang, J.S.; Kang, H.S.; Sohn, C.H.; Oh, C.W.; Kwon, O.K.; Kim, J.E. A retrospective analysis on the natural history of incidental small paraclinoid unruptured aneurysm. J. Neurol. Neurosurg Psychiatry 2014, 85, 289-294. [CrossRef] [PubMed] 
10. Aubreville, M.; Knipfer, C.; Oetter, N.; Jaremenko, C.; Rodner, E.; Denzler, J.; Bohr, C.; Neumann, H.; Stelzle, F.; Maier, A. Automatic classification of cancerous tissue in laserendomicroscopy images of the oral cavity using deep learning. Sci. Rep. 2017, 7, 11979. [CrossRef] [PubMed]

11. Esteva, A.; Kuprel, B.; Novoa, R.A.; Ko, J.; Swetter, S.M.; Blau, H.M.; Thrun, S. Dermatologist-level classification of skin cancer with deep neural networks. Nature 2017, 542, 115-118. [CrossRef] [PubMed]

12. Nakao, T.; Hanaoka, S.; Nomura, Y.; Sato, I.; Nemoto, M.; Miki, S.; Maeda, E.; Yoshikawa, T.; Hayashi, N.; Abe, O. Deep neural network-based computer-assisted detection of cerebral aneurysms in mr angiography. J. Magn. Reson. Imaging 2018, 47, 948-953. [CrossRef] [PubMed]

13. Stember, J.N.; Chang, P.; Stember, D.M.; Liu, M.; Grinband, J.; Filippi, C.G.; Meyers, P.; Jambawalikar, S. Convolutional neural networks for the detection and measurement of cerebral aneurysms on magnetic resonance angiography. J. Digit. Imaging 2018. [CrossRef] [PubMed]

14. McDowell, M.M.; Zhao, Y.; Kellner, C.P.; Barton, S.M.; Sussman, E.; Claassen, J.; Ducruet, A.F.; Connolly, E.S. Demographic and clinical predictors of multiple intracranial aneurysms in patients with subarachnoid hemorrhage. J. Neurosurg. 2018, 128, 961-968. [CrossRef]

15. Nehls, D.G.; Flom, R.A.; Carter, L.P.; Spetzler, R.F. Multiple intracranial aneurysms: Determining the site of rupture. J. Neurosurg. 1985, 63, 342-348. [CrossRef] [PubMed]

16. Hino, A.; Fujimoto, M.; Iwamoto, Y.; Yamaki, T.; Katsumori, T. False localization of rupture site in patients with multiple cerebral aneurysms and subarachnoid hemorrhage. Neurosurgery 2000, 46, 825-830. [PubMed]

17. Jeon, J.P.; Kim, C.; Oh, B.D.; Kim, S.J.; Kim, Y.S. Prediction of persistent hemodynamic depression after carotid angioplasty and stenting using artificial neural network model. Clin. Neurol. Neurosurg. 2018, 164, 127-131. [CrossRef] [PubMed]

18. Jeon, J.S.; Sheen, S.H.; Hwang, G.J.; Kim, H.C.; Kwon, B.J. Feasibility of intravenous flat panel detector ct angiography for intracranial arterial stenosis. AJNR Am. J. Neuroradiol. 2013, 34, 129-134. [CrossRef] [PubMed]

19. Jou, L.D.; Mohamed, A.; Lee, D.H.; Mawad, M.E. 3d rotational digital subtraction angiography may underestimate intracranial aneurysms: Findings from two basilar aneurysms. AJNR Am. J. Neuroradiol. 2007, 28, 1690-1692. [CrossRef]

20. Lu, L.; Zhang, L.J.; Poon, C.S.; Wu, S.Y.; Zhou, C.S.; Luo, S.; Wang, M.; Lu, G.M. Digital subtraction ct angiography for detection of intracranial aneurysms: Comparison with three-dimensional digital subtraction angiography. Radiology 2012, 262, 605-612. [CrossRef]

21. Sekhar, L.N.; Tariq, F.; Mai, J.C.; Kim, L.J.; Ghodke, B.; Hallam, D.K.; Bulsara, K.R. Unyielding progress: Treatment paradigms for giant aneurysms. Clin. Neurosurg. 2012, 59, 6-21. [CrossRef] [PubMed]

22. International Study of Unruptured Intracranial Aneurysms Investigators. Unruptured intracranial aneurysms-risk of rupture and risks of surgical intervention. N. Engl. J. Med. 1998, 339, 1725-1733. [CrossRef] [PubMed]

23. Wiebers, D.O.; Whisnant, J.P.; Huston, J., 3rd; Meissner, I.; Brown, R.D., Jr.; Piepgras, D.G.; Forbes, G.S.; Thielen, K.; Nichols, D.; O'Fallon, W.M.; et al. Unruptured intracranial aneurysms: Natural history, clinical outcome, and risks of surgical and endovascular treatment. Lancet 2003, 362, 103-110. [CrossRef]

24. Dolati, P.; Pittman, D.; Morrish, W.F.; Wong, J.; Sutherland, G.R. The frequency of subarachnoid hemorrhage from very small cerebral aneurysms ( $<5 \mathrm{~mm}$ ): A population-based study. Cureus 2015, 7, e279. [PubMed]

25. Williams, L.N.; Brown, R.D., Jr. Management of unruptured intracranial aneurysms. Neurol. Clin. Pract. 2013, 3, 99-108. [CrossRef]

26. Xiang, J.; Natarajan, S.K.; Tremmel, M.; Ma, D.; Mocco, J.; Hopkins, L.N.; Siddiqui, A.H.; Levy, E.I.; Meng, H. Hemodynamic-morphologic discriminants for intracranial aneurysm rupture. Stroke 2011, 42, $144-152$. [CrossRef]

27. Boussel, L.; Rayz, V.; McCulloch, C.; Martin, A.; Acevedo-Bolton, G.; Lawton, M.; Higashida, R.; Smith, W.S.; Young, W.L.; Saloner, D. Aneurysm growth occurs at region of low wall shear stress: Patient-specific correlation of hemodynamics and growth in a longitudinal study. Stroke 2008, 39, 2997-3002. [CrossRef] [PubMed]

28. Malek, A.M.; Alper, S.L.; Izumo, S. Hemodynamic shear stress and its role in atherosclerosis. JAMA 1999, 282, 2035-2042. [CrossRef] [PubMed] 
29. Hassan, T.; Timofeev, E.V.; Saito, T.; Shimizu, H.; Ezura, M.; Matsumoto, Y.; Takayama, K.; Tominaga, T.; Takahashi, A. A proposed parent vessel geometry-based categorization of saccular intracranial aneurysms: Computational flow dynamics analysis of the risk factors for lesion rupture. J. Neurosurg. 2005, 103, 662-680. [CrossRef] [PubMed]

30. Shojima, M.; Oshima, M.; Takagi, K.; Torii, R.; Hayakawa, M.; Katada, K.; Morita, A.; Kirino, T. Magnitude and role of wall shear stress on cerebral aneurysm: Computational fluid dynamic study of 20 middle cerebral artery aneurysms. Stroke 2004, 35, 2500-2505. [CrossRef] [PubMed]

31. Laukamp, K.R.; Thiele, F.; Shakirin, G.; Zopfs, D.; Faymonville, A.; Timmer, M.; Maintz, D.; Perkuhn, M.; Borggrefe, J. Fully automated detection and segmentation of meningiomas using deep learning on routine multiparametric MRI. Eur. Radiol. 2019, 29, 124-132. [CrossRef] [PubMed]

32. Sailer, A.M.; Wagemans, B.A.; Nelemans, P.J.; de Graaf, R.; van Zwam, W.H. Diagnosing intracranial aneurysms with $\mathrm{mr}$ angiography: Systematic review and meta-analysis. Stroke 2014, 45, 119-126. [CrossRef] [PubMed]

33. Sugahara, T.; Korogi, Y.; Nakashima, K.; Hamatake, S.; Honda, S.; Takahashi, M. Comparison of 2d and 3d digital subtraction angiography in evaluation of intracranial aneurysms. AJNR Am. J. Neuroradiol. 2002, 23, 1545-1552.

34. Tykocki, T.; Kostkiewicz, B. Aneurysms of the anterior and posterior cerebral circulation: Comparison of the morphometric features. Acta Neurochir. (Wien) 2014, 156, 1647-1654. [CrossRef] [PubMed]

35. Yu, W.; Yang, K.; Bai, Y.; Yao, H.; Rui, Y. Visualizing and comparing AlexNet and VGG using deconvolutional layers. In Proceedings of the 33rd International Conference on Machine Learning, New York, NY, USA, 19-24 June 2016.

(C) 2019 by the authors. Licensee MDPI, Basel, Switzerland. This article is an open access article distributed under the terms and conditions of the Creative Commons Attribution (CC BY) license (http://creativecommons.org/licenses/by/4.0/). 